\title{
Lessons Learned from the Wide Field Camera 3 Flight Correlation
}

\author{
Hume L. Peabody ${ }^{1}$, Richard A. Stavely ${ }^{1}$, and Jackie Townsend ${ }^{2}$ \\ NASA Goddard Space Flight Center, Greenbelt MD 20771 \\ Josh $\mathrm{Abel}^{3}$ and Joe Mandi ${ }^{3}$ \\ Lockheed Martin IS and GS, Greenbelt, MD 20771 \\ and \\ William Bast ${ }^{4}$ \\ Alliant Tech Systems, Beltsville, MD 20705
}

The Wide Field Camera 3 (WFC3) instrument was installed into the Hubble Space Telescope as part of the activities for STS-125 (HST Servicing Mission 4). Initial model predictions for power and radiator temperature were not in good agreement with flight data during a relatively hot, stable period, with the flight power and temperatures being significantly higher than predictions. Significant efforts were undertaken to identify the causes of the discrepancies and to resolve the flight model correlation problems as the thermal vacuum test correlation indicated good agreement. The WFC3 thermal design performance has proven difficult to accurately predict, since the power dissipation on the radiator typically increases as the radiator temperature increases, due to a Thermo Electric Cooler (TEC) attached to the this radiator. This self heating continues until the radiative emissive capability is met for a given temperature, and only then does the system find a quasi-steady regime. Various other factors may also contribute to the radiator temperature, such as backloading from the observatory itself and the planet, local high absorptivity regions near fasteners/holes, and temperature varying parasitic heat leaks from the instrument itself to the radiator. Each of these effects in turn may increase the radiator temperature, and furthermore the demand on the TEC.

\section{Nomenclature}

$\begin{array}{ll}H S T & =\text { Hubble Space Telescope } \\ W F / P C I I & =\text { Wide Field Planetary Camera II } \\ W F C 3 & =\text { Wide Filed Camera } 3 \\ T V & =\text { Thermal Vacuum } \\ T E C & =\text { Thermo Electric Cooler } \\ G C H P & =\text { Gas Charged Heat Pipe } \\ V C H P & =\text { Variable Conductance Heat Pipe } \\ N C G & =\text { Non Condensable Gas } \\ U V I S & =\text { Ultraviolet VISible } \\ M L I & =\text { Multi-Layer Insulation } \\ C C D & =\text { Charge Coupled Device } \\ I R & =\text { InfraRed } \\ F P A & =\text { Focal Plane Assembly } \\ T E C F I R E & =\text { Thermo Electric Cooling For InfraRed Electronics } \\ D B & =\text { Detector Baseplate }\end{array}$

${ }^{1}$ Thermal Engineer, Thermal Engineering Branch, Code 545 Goddard Space Flight Center, Greenbelt MD 20771

${ }^{2}$ Advanced Mission Concepts Manager, Astrophysics Projects Division, Code 440, Goddard Space Flight Center, Greenbelt MD 20771

${ }^{3}$ Thermal Engineer, Lockheed Martin IS\&GS, 7474 Greenway Center Dr, Greenbelt MD 20770

${ }^{4}$ Thermal Engineer, ATK Aerospace Systems Group, 5050 Powder Mill Rd, Beltsville MD 20705 


$\begin{array}{ll}C E & =\text { Cold Enclosure } \\ L V P S & =\text { Low Voltage Power Supply } \\ M E B & =\text { Main Electronics Box } \\ D E B & =\text { Detector Electronics Box } \\ C E B & =\text { CCD Electronics Box }\end{array}$

\section{Introduction}

$\mathbf{T}$ The Wide Field Camera 3 (WFC3) instrument replaced the Wide Field Planetary Camera II instrument (WF/PCII) on the Hubble Space Telescope (HST). The instrument consists of two channels: UV/Visible (UVIS) and Infrared (IR). A pickoff mirror reflects the incoming light to a channel select mechanism, which then directs the light through either the UVIS path or the IR path to the corresponding detector assembly. Both the UVIS and IR detector subsystems utilize multi-stage Thermo Electric Coolers (TECs) in several ways. TECs are used to directly cool the IR focal plane array (FPA) and the UVIS charged coupled device (CCD) and to control the surrounding shields/housings to isolate the detector assemblies from the rest of the instrument. The instrument has two main radiators, one to reject the majority of the electronics heat and one to reject the detector heat. Furthermore, some of the lower dissipating electronics boxes reject their heat directly to the HST interior.

After near cancellation due to suspension of Shuttle flights after the Columbia accident, sufficient resources were available for a characterization Thermal Vacuum test, referred to as TV1. Later, the program was revived and WFC3 successfully completed two further thermal vacuum tests, TV2 and TV3. TV2 was aimed at qualifying the instrument and subsystems for flight. TV3 was used to calibrate and test the final flight detectors which had higher performance capabilities than those that were tested during TV2. WFC3 was installed into the Hubble Space Telescope in May 2009 and has performed very well. However, the initial analytical predictions of the thermal performance of the instrument were not in good agreement with flight data. Therefore, a flight correlation was performed to understand the discrepancies between model predictions and flight performance.

\section{WFC3 Thermal Design}

Figure 1 shows the general thermal design for WFC3. WFC3 consists of two actively cooled detectors (UVIS $\mathrm{CCD}$ and IR focal plane array), housed inside of temperature controlled assemblies. These assemblies are mounted inside a thermally controlled Optical Bench. The bench is structurally tied to the HST frame via low conductivity struts which connects to three thermally controlled latch points. The bench itself is thermally controlled by the Cold Plate mounted below the bench, which is maintained at temperature by a combination of heaters and a Variable Conductance Heat Pipe (VCHP). The entire Optical Bench and Cold Plate subsystems are radiatively isolated from

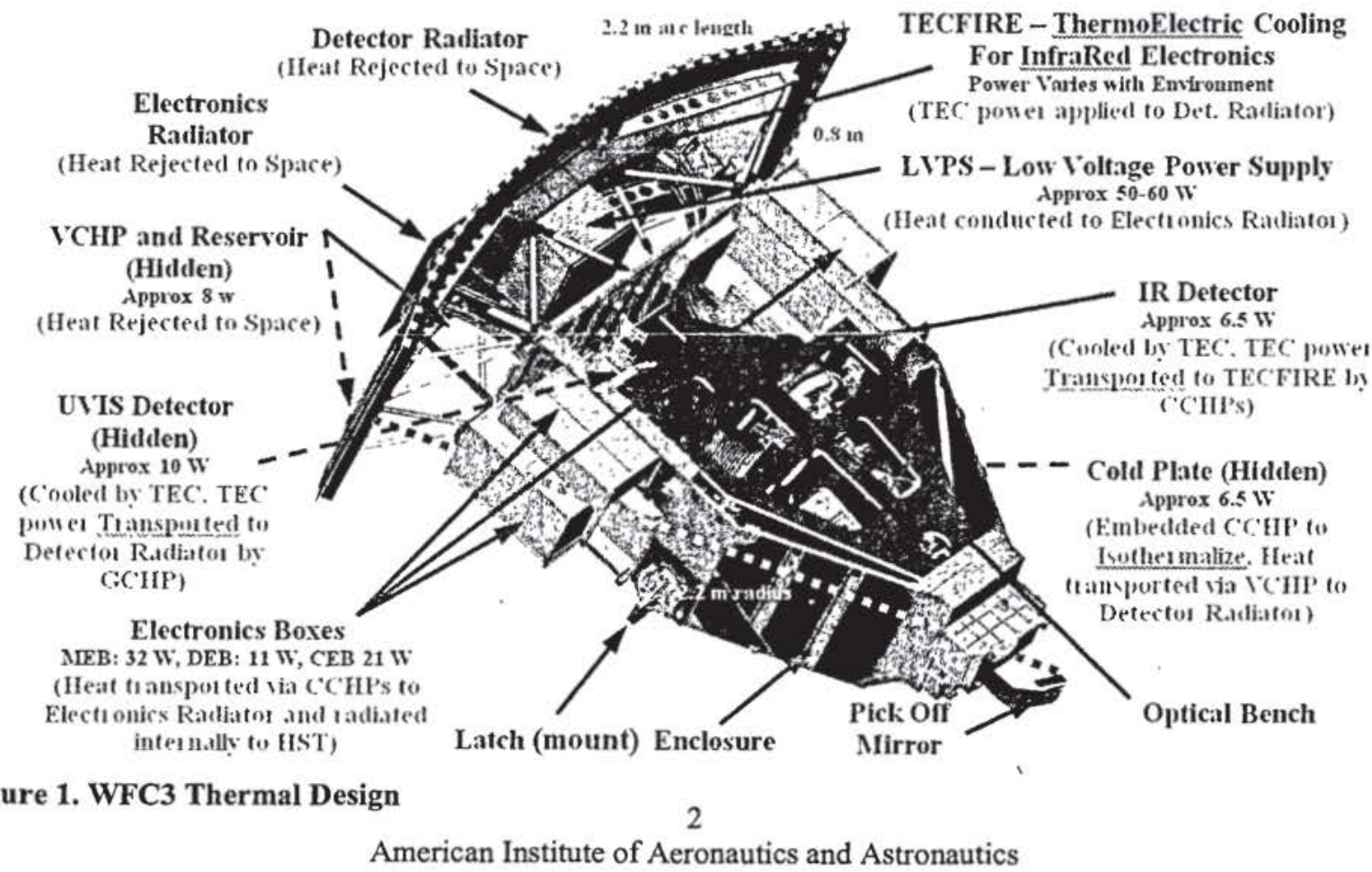


the surroundings by multi-layer insulation (MLI). Furthermore, the surrounding MLI is enclosed in an exoskeleton panel configuration to which the external electronics boxes are mounted. Two radiators are employed to reject the majority of the power dissipation to space: the Detector Radiator and the Electronics Radiator.

The Detector Radiator includes a network of six spreader heat pipes perpendicular to two header heat pipes (Upper and Lower) to help isothermalize the radiator and increase its efficiency. Figure 2 shows the detector radiator (with the electronics radiator removed) and the associated heat pipe network. A number of transport heat pipes are used to move the heat from dissipation locations within the instrument to dedicated locations on the radiators. Four major heat paths to the radiators exist: UVIS Detector, IR Detector, Optical Bench/Cold Plate, and the Main Electronics Boxes.

The UVIS detector is a Charge Coupled

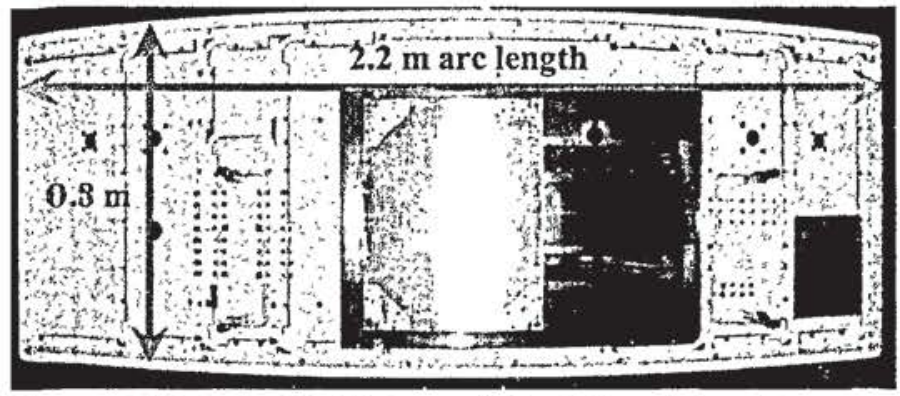

Figure 2. Detector Radiator and Heat Pipe Network

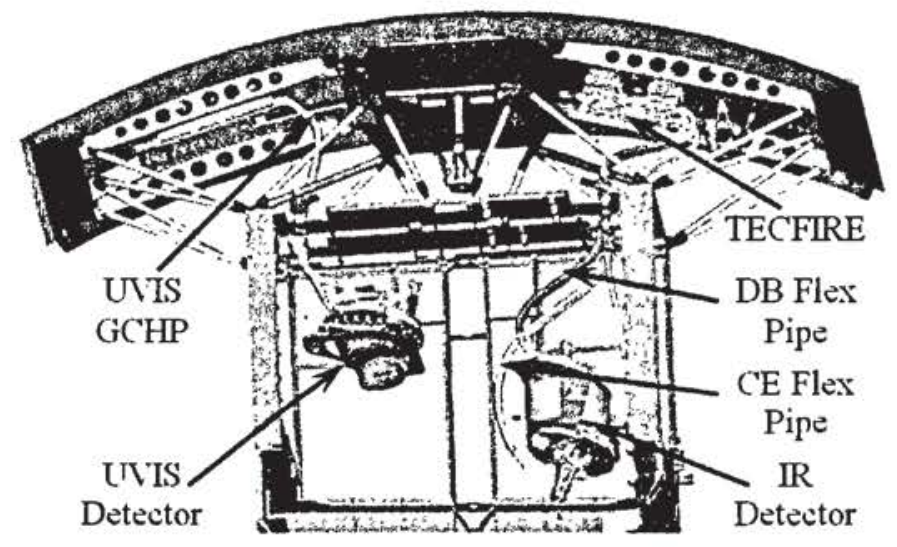

Figure 3. WFC3 Detector HeatPipe Thermal control

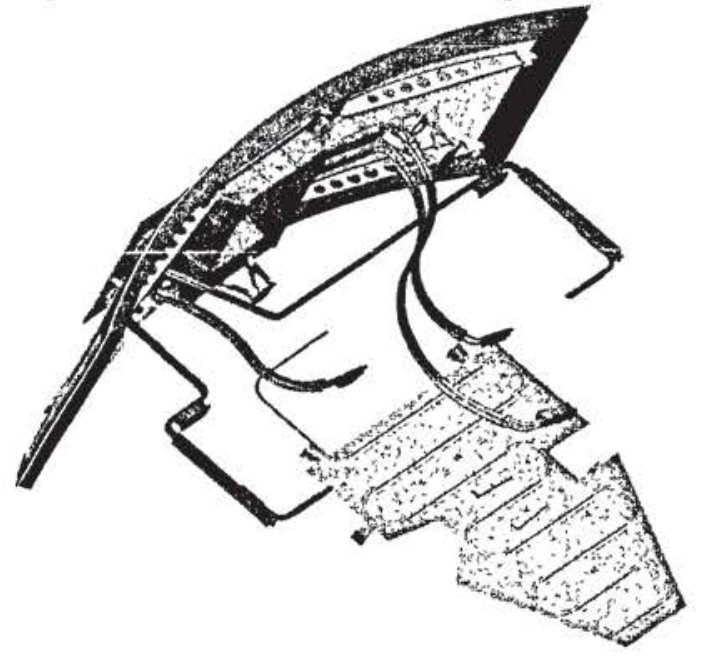

Figure 4. WFC3 HeatPipe Layout Device (CCD) which is actively cooled by a 4stage Thermo-Electric Cooler (TEC) to $-83^{\circ} \mathrm{C}$. Surrounding the UVIS detector is a radiative isolation shield which is cooled by four constant current, 2-stage TECs. The heat from all of these TECs is deposited on the UVIS detector baseplate, which is conductively coupled to the evaporator end of a Gas Charged Heat Pipe (GCHP). The condenser end of the GCHP is coupled directly to the Detector Radiator which is used to reject its heat to space. Figure 3 shows the UVIS detector thermal control system, as well as the thermal control system for the IR detector.

Similarly, the IR detector consists of a Focal Plane Array (FPA) which is actively cooled by a 6-stage TEC to $-128^{\circ} \mathrm{C}$. The IR detector assembly is housed inside a Cold Enclosure which provides a layer of isolation between the detector and the surrounding instrument surfaces. The heat from the 6-stage TEC is deposited on the detector assembly baseplate, which is conductively coupled to the evaporator end of a flexible heat pipe. Furthermore, the top of the Cold Enclosure is also coupled to the evaporator end of a second flexible heat pipe. Instead of coupling these two heat pipes condensers directly to the temperature varying radiator, they are coupled to an interfacing assembly called TECFIRE: Thermo-Electric Cooling For InfraRed Electronics. TECFIRE consists of 3 units, each of which contains a $3 \times 2$ array of 1-stage TECs in parallel (two units for the detector baseplate: DB1 and DB2, and one unit for the Cold Enclosure: CE). TECFIRE provides a thermally stable heat sink for the IR heat pipe condensers, nominally maintaining $-53^{\circ} \mathrm{C}$ for the $\mathrm{DB}$ and $-45^{\circ} \mathrm{C}$ for the $\mathrm{CE}$ using commandable setpoints. The heat removed by the TECs, as well as the power generated by the TECs, is deposited directly on the Detector Radiator and rejected to space.

Both detectors reside inside the Optical Bench whose bulk temperature is nominally maintained at $0^{\circ} \mathrm{C}$ by the Cold Plate below the Optical Bench. The Cold Plate contains a constant 
conductance heat pipe (CCHP) embedded in the panel and a heater to help provide an isothermal, warm sink to regulate the Optical Bench temperature, as shown in Figure 4. Furthermore, the condenser end of the Cold Plate $\mathrm{CCHP}$ is coupled to the evaporator end of a VCHP, with the VCHP condenser coupled directly to the detector radiator. For these two heatpipes in series, the VCHP is used provide stability by minimizing the impact of the varying detector radiator temperature.

Lastly, four major electronics boxes reject their heat to the Electronics Radiator or directly to the internal HST surfaces. Two Main Electronic Boxes (MEB, nominally $32 \mathrm{~W}$ ) are coupled to the Electronics Radiator via individual CCHPs and are coated with a low emissivity coating to minimize their radiative heat loss/gain from the internal HST surfaces. The Low Voltage Power Supply (LVPS) is mounted directly to the back side of the Electronics Radiator, and rejects its heat to space via the Electronics Radiator. The Detector Electronics Box (DEB, nominally $11 \mathrm{~W}$ ) and the CCD Electronic Box (CEB, nominally $21 \mathrm{~W}$ ) are coated with high emissivity coatings and are intended to reject their heat directly to the internal HST components.

One challenging aspect of the design is that as the Detector Radiator temperature increases, the detector temperature control power also increases. This is contrary to conventional spacecraft where the power demand typically decreases when heat sink temperatures increase. This is a by-product of the extensive use of TECs throughout the design and makes predictions of the design behavior a bit more difficult.

\section{Instrument and Subsystem Testing}

\section{A. TV3 Correlation}

After the TV2 test campaign in 2007 and the corresponding correlation, significant efforts were made to understand the heat leaks to the radiator as the radiator was much warmer in test than predicted by the model. Various heat leak sources were suspected including: worse than expected blankets, vents line, vent line supports, test and flight instrumentation wires, etc. However, test data available from TV2 could neither support nor counter all of these hypotheses. The correlation effort, for lack of a better solution, made some adjustments to the model that could not necessarily be well supported (MLI $\mathrm{e}^{*}$ values, adjustment of emissivity to account for effective radiating area, etc.) in order to diminish the ability of the radiator to reject heat, consequently making it warmer.

Fortunately, the TV3 test with the final flight detectors provided a better opportunity to quantify these heat leaks and to make minor design changes to minimize the heat leak associated with the vent line supports by installing a blanket to cover these assemblies. Additional test instrumentation was added to quantify each of these heat leak paths so that the TV3 correlation could better justify some of the model updates and eliminate some of the changes that could no longer be supported by the test data. This test provided an invaluable opportunity to gain substantive data to corroborate or eliminate the changes made to correlate the model to TV2 test data. All changes were made in models representing both the TV2 and TV3 configurations to ensure that no model updates were in conflict with either set of test data.

- Additional test opportunities can be extremely valuable since additional information can be gained from areas using additional test instrumentation that were not known ahead of time to have large uncertainties.

- Model changes made during a correlation effort should never invalidate the correlation to test data from previous testing for regions where the design was not significantly varied.

\section{B. Sufficient Subsystem Testing}

One area of larger uncertainty was the TECFIRE power which is greatly amplified by either more heat needing to be removed or more heat on the hot side resulting in a larger DT to overcome. Fortunately, the TECFIRE requalification test, well before the TV2 and TV3 tests, provided an opportunity to verify the performance curves from the TEC vendor and establish confidence in predictions made for TECFIRE. At the request of the thermal subsystem, numerous combinations of parasitic heat input, hot side temperature, cold side temperature, and TEC power were tested to generate points to compare against the vendor supplied data. Knowing three of the four parameters allows for solution (iteratively or directly) of the fourth. The thermal model predicts TEC current and voltage (i.e. power) from input parasitic heat, hot side and cold side temperatures as predicted by the thermal model. Data was collected for a total of 43 cases with the current and voltage needed to maintain the steady conditions with known hot and cold side temperatures and parasitic heat inputs compared to analytical predictions. The results showed a very good correlation for the prediction of the achievable temperature difference versus input current. However, the TEC voltage as a function of current relations needed slight adjustment. 
At numerous points throughout the program, this data has proven extremely useful as an anchor point to justify the continued use of the data in offline and empirical models. A key use of the verified vendor performance curves was the estimation of the heat being removed by the TEC to be computed, which is a parameter not readily available from the telemetry stream. This heat removal calculation made it possible to quantify any additional parasitics added to the TECFIRE flex heatpipes that may not be accounted for in the model, since the upstream IR detector power is known. The difference between the amount of heat removed by the DB TECFIRE and the IR FPA TEC power is an additional parasitic added to the model as a fixed parameter not included in the current fidelity of the detailed model.

- Subsystem qualification tests, particularly for thermally sensitive subsystems, can provide a great opportunity for the thermal engineer to gain additional data that may provide confidence in hand or empirical checks at later points in a program.

\section{Flight Correlation}

The WFC3 instrument was installed into the Hubble Space Telescope on May 14, 2009 as part of the 4th Servicing Mission, also known as STS-125. After initial checkout of the instrument, the detectors were cooled down to their nominal operating temperatures. Initial power levels for TECFIRE were much higher in flight than anticipated based on model predictions. This caused concern, since the initial post SM4 attitudes were not expected to reflect the most extreme attitude and environmental parameters. A better understanding of the TECFIRE behavior was pursued, focusing on power stability and the prevention of TEC run-away. Modeling assumptions and inputs throughout the model were revisited to provide better predictions of the flight conditions while still maintaining good correlation with the TV test data.

Initial comparisons showed that the detector radiator predicted about $11^{\circ} \mathrm{C}$ colder than flight for operational cases with predicted instrument power being about $2.3 \mathrm{~W}$ less (compared to a nominal power of approximately 200 W total). The Protect Operate mode, which represented a fairly different power configuration with the detectors not being cooled, predicted about $6^{\circ} \mathrm{C}$ colder for the radiator with $10.5 \mathrm{~W}$ more instrument power predicted than actual. Even when the operational power values from telemetry were input into the model as known inputs, the detector radiator predicted about $7^{\circ} \mathrm{C}$ colder than flight values. For these cases, it was revealed that significant uncertainties still existed in the heat paths to and from the detector radiator. Efforts were focused on: (1) parasitic heat leaks from the warm portion of the instrument (especially for the TECs), (2) environmental heating, (3) effective radiating area, and (4) backloading from HST.

The overall driving concern was whether the instrument could maintain the detectors at their required cold temperatures under the mission extreme hot environmental conditions. Based on the much higher temperatures than anticipated seen initially in flight, confidence needed to be established in the thermal model as a useful tool for predicting the performance of the instrument under more extreme conditions as well as identifying what set of adjustable control parameters might yield optimum thermal performance.

All modeling changes for flight correlation were also run for TV2 and TV3 correlation as the TV data serves as additional anchor points for the validity of the model. While some of the parameters may have produced a good correlation to test data, they may not have been tuned well enough for flight. One such example was the gas charge for the GCHP controlling the UVIS detector. The UVIS power during test correlated well with the current gas charge, but not well for flight. Increasing this charge resulted in good correlation for flight while maintaining the correlation to test data and highlighted the sensitivity of the predictions to this parameter.

\section{A. Understanding of Design Behavior}

Based on TV2 and TV3 experiences, the radiative sink for TECFIRE needed to be closely monitored, since too warm of a sink could cause an eventual run-away condition for TECFIRE. The run-away condition is caused when an increase in TEC power results in a net increase in the TEC cold side temperature. When an increase in TEC power is applied, two things happen: (1) the temperature difference between the hot side and cold side of the TEC increases and (2) the increased electrical power results in a warmer hot side when connected to a finite sink. The net result therefore may be a larger increase in hot side temperature than the decrease in the hot-cold DT and consequently, a net increase in the cold side temperature. This phenomenon was well characterized prior to the TV2 test and documented in Reference 3.

Figure 5 shows the achievable cold side temperature for a given TEC current and radiative sink. Each curve has a maximum current before reaching a condition where increasing the current results in an increase in the cold side temperature instead of a decrease; this point is deemed the power breakaway current. Once the power breakaway current is exceeded, continuous increasing of the current is inevitable based on the WFC3 controller design unless the sink temperature is reduced or the input source heat load changes. If the sink is insufficient to allow the goal 
temperature to be achieved, then only a current limiting device on the hardware will constrain the current to the TEC.

This phenomenon leads to the next logical question of "How can a steady condition ever be reached that is not based on a hardware current limiting device if the power breakaway current is exceeded?" If the current and power

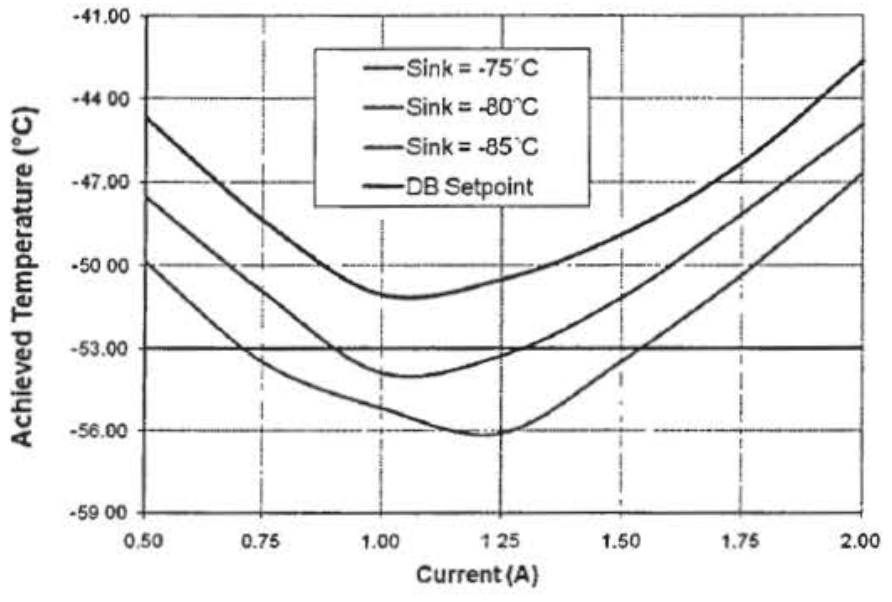

Figure 5 - Achievable Cold Temperature for Given Sink and fixed TEC Current continuously increase past this point, why would the temperature of the hot side reach steady state for a fixed sink temperature? The answer is based on the non-linearity of radiative heat transfer. As the heat rejection temperature increases $(\mathrm{T}+\mathrm{dT})$, the amount of heat that can be rejected to a constant sink $(\mathrm{Q}+\mathrm{dQ})$ increases at a faster rate. $\left(\mathrm{dQ} / \mathrm{dT} \alpha 4 \mathrm{~T}^{3}\right.$ ). Eventually, the amount of heat that can be rejected balances the amount of heat generated by the TEC and a steady condition is reached. Whether this occurs at a current lower than the hardware limitation is a matter of the design. Examining Figure 5 shows two balanced points for low enough sink temperatures, both of which are perfectly valid stable operating points. Which point is selected by the controller depends on the previous values

- For actively cooled systems, the characterization of all heat paths to the hot side, especially environmental heat loads, is of crucial importance to being able to accurately predict a steady condition.

\section{B. Flight vs. Test Correlation Parameters}

Various uncertainty parameters from the TV3 correlation efforts were also revisited. This mainly included additional parasitic heat loads absorbed by the heat pipes that transported the heat to TECFIRE. These heat loads were estimated based on knowing the TEC hot and cold side temperatures and the TEC current for an achieved condition from test data. Based on the performance curves, the heat removed by the TEC could be computed and compared to measured IR detector power values upstream from TECFIRE. The differences between the calculated heat removed by the TEC and the measured IR detector power was the additional load not captured by the model and was assumed to be fairly constant given the stable conditions of the HST interior. Since the values used in test are based on the sink temperatures of the side cryo panels, the possibility exists that the flight heat loads may be different if the flight HST interior sinks was different than the test cryo panel temperatures.

- Once a test correlation is complete, some benefit may be derived by performing a sensitivity study on the tuned parameters. This could identify the range to which these may be further altered for flight correlation without negatively impacting a test correlation.

\section{Environmental Parameters}

The initial values used for flight correlation for Earth IR, Solar, and Albedo heat fluxes were the average of the design maximum and minimum values. While this was the simplest approach, these average values may not accurately represent the true fluxes at a specific flight data point. For a design such as WFC3, where the TEC power and radiator temperatures are very sensitive to the environmental sinks, errors in these parameters could cause large discrepancies in the model predictions when compared to flight data. The spacecraft contractor investigated the accuracy of using average environmental flux values and found that much higher Earth IR fluxes are typical for the latitudes between which HST orbits.

Therefore, for any designs that are highly dependent on the environmental sinks, it is strongly recommended that the appropriate environmental parameters are investigated and justified. No matter how good a model is, if it is not simulated with the correct boundary conditions, it will not predict the behavior as seen in test or flight.

- When correlating to flight data, care must be taken when selecting the environmental parameters to use. While extreme bounding cases may be the simple choice, they may not be accurate for a specific set of conditions related to flight. 


\section{- If a design is very sensitive to environmental parameters and a flight correlation is essential, it may be beneficial to include some means of measuring the environmental fluxes at the radiator in flight.}

\section{LOCALIZED OPTICAL PROPERTY EFFECTS}

The detector radiator itself was modeled as a continuous, smooth surface with pristine optical properties measured in an area with relatively no surface irregularities. In actuality, the radiator has numerous holes, cap nuts, and features that could locally affect the optical properties in this region. Furthermore, most of these features are at interfaces to important hardware components

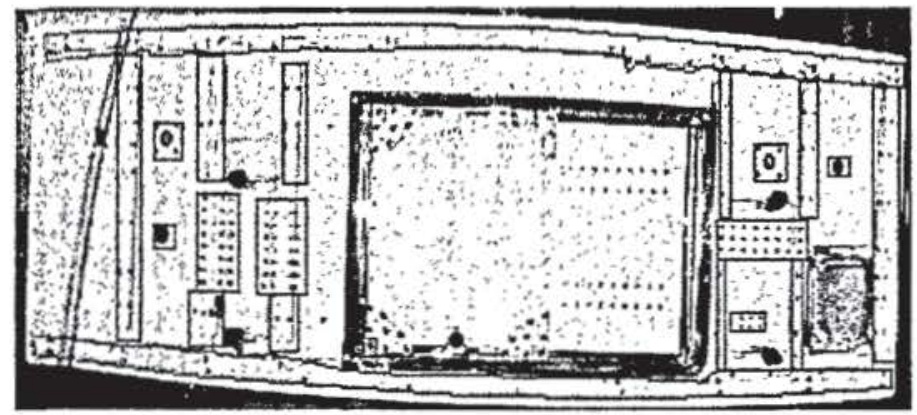

Figure 6. Detector Radiator Image used for Estimation of Localized Optical Property Effects (heat pipes, valve brackets, etc.) where the telemetry is being read. A unique approach was taken to evaluate a high fidelity picture of the radiator using contamination particle counting techniques to determine the percentage of each region that would be considered non-pristine. This percentage would likely have a much higher absorbed albedo flux (with the features acting as light traps). Conversely, the irregular areas would likely not have as good a local emissive capability due to their inherent lower emissivity and the thermal contact resistance between the radiating surfaces and the inefficient radiating areas.

Figure 6 shows the picture with the regions highlighted in red that were evaluated. The portion in the center of the image is the Electronics Radiator, which is independent of the Detector Radiator and consequently was not evaluated. Similarly, the small VCHP reservoir radiator in the lower right comer was also not evaluated. Based on the results of the imaging, percentage dark values were computed for each region and localized adjusted optical properties applied to the affected surfaces. The optical properties were based on:

$$
\begin{aligned}
& \alpha_{\text {eff }}=\%_{\text {dark }} * 1.0+\left(1-\%_{\text {dark }}\right) * \alpha_{\text {WhitePaint }} \\
& \varepsilon_{\text {eff }}=\%_{\text {dark }} * 0.5+\left(1-\%_{\text {dark }}\right) * \varepsilon_{\text {WhitePaint }}
\end{aligned}
$$

The application of these localized properties increased the corresponding nodal temperatures (higher absorptivity, lower emissivity) of locations more closely tied to the telemetry points without necessarily raising the overall temperature of the radiator. In essence, it was judged that the overall heat rejected by the radiator was correct, but direct use of temperatures at localized areas of inefficiency as being representative of the entire radiator temperature may not have been the best approach.

- Localized optical property effects (holes, cap nuts, etc.) due to mechanical features may artificially influence the sensed temperature values from telemetry. These effects should be considered when temperature data is compared to model predictions.

\section{E. MODEL COMPLETENESS}

The Detector Radiator tends to run fairly cold ( -40 to $-50 \mathrm{C}$ range), and at this cold temperature, the backloading from other warmer spacecraft surfaces may be important. The radiator has a fairly clear field of view to space, but one major component of considerable size in the field of view is the main reflector and mast of the High Gain Antenna (HGA). This component was not previously included in the model as it had no telemetry and the previous instrument (WF/PC II) did not show any adverse effect to its absence in the model. However, once the HGA dish and mast were added in to the model, it did highlight some warm conditions where it could get hot and provide a non trivial backload onto the WFC3 radiator. This accounted for about 1 extra W of heating on the Detector Radiator, which may be further amplified by TEFIRE demands.

- Although some surfaces may not be included in a flight model due to a lack of telemetry or insight, their backloading effect on other surfaces should be considered before excluding them from the model.

\section{Empirical Model}

An empirical model was developed based on performing an energy balance on the Detector Radiator using flight data. The purpose of this model was three-fold: (1) to perform a sanity check on the flight data, (2) to allow quick 
determination of sensitivity to various uncertainties and parameters, and (3) to allow possible prediction of instrument performance based on Detector Radiator temperature. Some assumptions were made in establishing this empirical model, namely that the radiator was at a single, uniform temperature, and that the area and optical properties were known properties from testing or evaluation. An energy balance for the Detector Radiator is shown

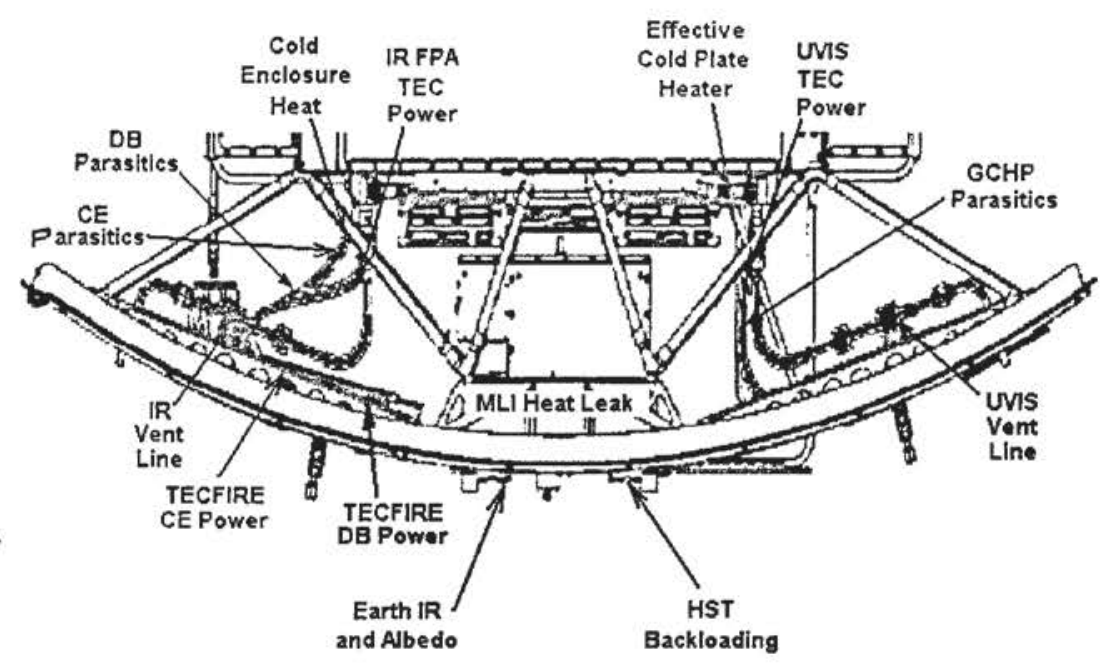

Figure 7. WFC3 Detector Radiator Energy Balance

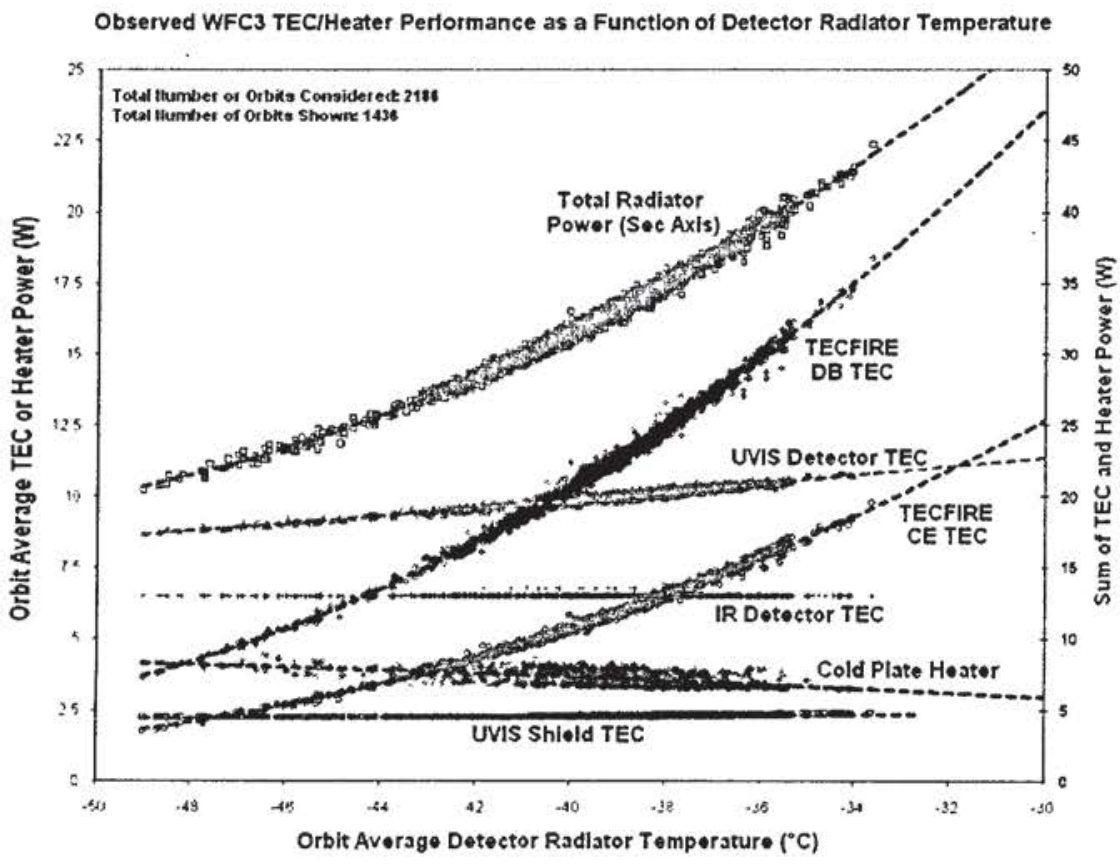

Figure 8. Flight Powers vs. Detector Radiator Temperature for Components Connected to Detector Radiator were also derived from TV test data including the heat removed by the CE TECFIRE unit, additional parasitic heat leaks absorbed by the flexible heatpipes for TECFIRE and the GCHP, as well as the estimated portion of Cold Plate heater power that reached the radiator as opposed to leaked out other paths. These values were considered constant and would not be greatly affected by internal effects.

The largest uncertainties were on the environmental flux (Earth IR and Albedo) and backloading onto the radiator from HST itself, due to radiative heat exchange with the Solar Arrays and High Gain Antenna (HGA). Using a geometric math model analyzed using ThermalDesktop, the solar array and HGA impacts were calculated

The empirical model first focused on known parameters which were extracted from the telemetry stream including: TECFIRE power, UVIS Detector and Shield TEC Power, IR FPA TEC power, and Cold Plate Heater Power. This data was reduced to represent an orbit average value to remove the effects of energy storage effects (mCp). Values over an entire orbit were averaged and criteria were applied to eliminate values where the orbiter was slewing or moving to a point of being unable to compute a reasonably stable orbit average. Of 2186 orbits evaluated, 1436 were suitable for use in the empirical analysis. Figure 8 shows these data points trend exists between the power value of each component and the radiator temperature. This data could be extrapolated to predict beyond the range of temperatures already experienced on flight with the caveat that the error may be larger.

Parameters with additional uncertainty were addressed next. Uncertainty in the effective radiating area (due to holes, cap nuts, heat pipes, etc.) and optical properties were investigated and values were used which were derived from the TV2 and TV3 test data and further refined based on the investigation into localized optical property effects. Further heat leaks to the

Figure 7. . 
throughout the allowable sun incidence angles. To estimate the earth IR and albedo for each orbit, however, it was ne cessary to calculate the earth view of the radiator at the subsolar point and 9AM/PM LST points. This calculation was compared with the output of the geometry model for several test orbits with good agreement.

\section{Total Radiator Power (Electronics + Environment) vs. $\sigma \mathrm{T}^{4}$ Averase Rasace}

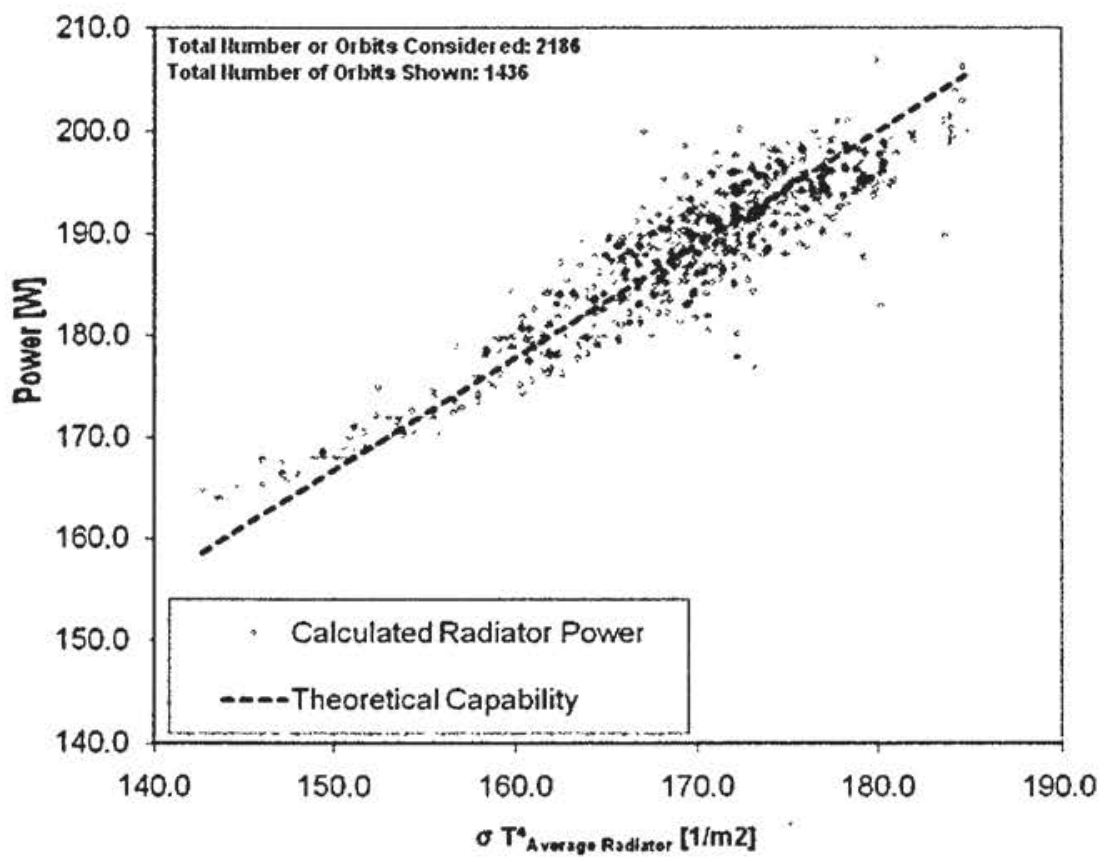

Figure 9. Actual Heat Rejection vs. Theoretical Heat Rejection based on Detector Radiator Temperature
Finally, it was noted that there was still an apparent periodic error in the calculation of the environmental heat load. After some research, the latitude and longitude of the vehicle at the subsolar point and 9AM/PM LST was used to estimate the variation in albedo and earth IR over the earth's surface. This modification fit well with the observed data. Using this method, the environmental heat load could be estimated for each orbit directly based on available ground system data.

One of the key benefits of developing this empirical model was the ability to very quickly determine radiator temperature sensitivity to numerous parameters such as instrument power, parasitic leaks, and environmental loads without the need to run a full orbital detailed thermal analysis. This allowed focus to be placed on the parameter uncertainties that resulted in the largest changes in radiator temperatures.

Taking the effective radiative capability of the radiator to space, where $A_{\text {eff }}=Q / \sigma\left(T_{r a d}{ }^{4}\right)$ and $Q$ includes all sources including instrument heat flows as well as environmental backloading, the value should be constant regardless of power or temperature. Figure 9 shows the variation in this effective area for all the data points and the trend reveals the error associated with the approach. Assuming that the entire scatter is due to uncertainty in the environmental heating, a histogram shows a normal distribution centered around zero with a standard deviation of about $3.3 \mathrm{~W}$. Two major components may contribute to this error: (1) uncertainty in albedo or Earth IR flux values and (2) a mid-orbit slew or maneuver which was not accounted for in the estimation. But given the large computational resources that would be necessary if each of these orbits and corresponding slew maneuvers was modeled in an analytical tool, the error is judged to be fairly small compared to the effort involved.

Using the observed fixed relationship between radiator temperature and the individual instrument heat loads, along with the estimation of the environmental and self heating from the spacecraft attitude, a predictive model was created. This model assumed the environment and iterated on radiator temperature until the heat inputs matched the radiating capability to space. As a check, this "predicted" temperature was compared against the actual temperature observed on orbit for all available flight data. While the errors in predicted temperature averaged close to zero, the inherent errors in the knowledge of the environment were amplified by the feedback on TEC demand. For example, if the assumed environment was $3 \mathrm{~W}$ higher than the true value, the temperature prediction at the radiator would reflect this error plus the error from the associated increased TEC demand. Conversely, if the assumed environment was $3 \mathrm{~W}$ lower than the true value, the TEC demand would also be lower. The resulting temperature predictions could be as much as $8 \mathrm{C}$ from their true value. By examining the empirical model performance, the thermal math model correlation was judged to be as accurate as possible given the reasonable uncertainty in the environmental heating parameters.

- Simplistic empirical models based on flight data and energy balances can be a valuable tool to cross check more computationally demanding detailed thermal models. 


\section{Future Predictions}

With a fairly well correlated model that agreed with both flight telemetry and ground testing telemetry and test data, the model could be confidently exercised to predict behavior under worst case conditions. In addition, the model can be used to evaluate the effects on instrument thermal control performance as a result of changing setpoints. Two key setpoints for possible adjustment to reduce the power demands are the TECFIRE DB and CE control temperatures. Increasing either or both of these setpoints reduces the associated cooling power, but also increases the IR FPA TEC cooling power to maintain the required IR FPA temperatures. The combined net effect of these cooling power changes may be a reduction in total power for the detector radiator. This setpoint adjustment was tested in TV3 and shown to reduce the overall power for the DB TEC system setpoint, but the change was not implemented for flight due to the large quantity of detector calibration data at the current setpoints. It should be noted that the IR FPA detector temperature would be the same between the two cases, as the IR FPA TEC setpoint was unchanged. While the current setpoints remain for flight, the capability exists to reduce the overall power demand if the design has insufficient margin with the current setpoints.

Figure 10 shows prediction for major TEC power sources and the detector radiator temperature nearest to TECFIRE predictions for the baseline control setpoints on the correlation day. While the model tends to over predict the TEC power, it does present a conservative case for the hot concerns. Since the actual IR fluxes are not known, the model may be slightly biased to the warm end, thereby over predicting the TEC powers. Figure 11 shows the predictions for worst case using the same control parameters. Using the model, adjustments to the control setpoints were simulated and the results are shown in Figure 12.

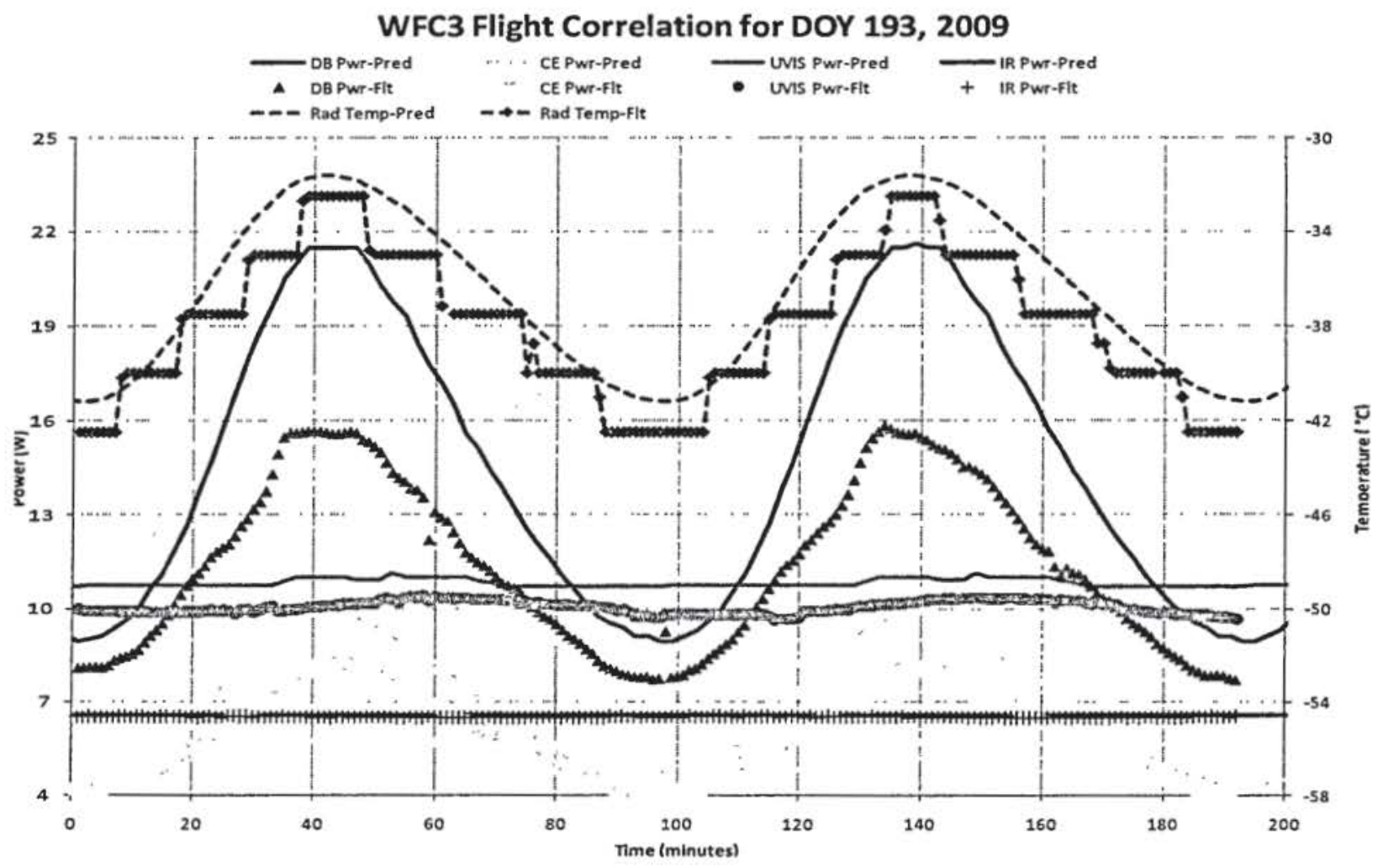

Figure 10 - Model Predictions vs. Flight for DOY 193

$<$ Still working out kinks in model, Will be added for final submission >

Figure 11 - TECFIRE Prediction for Hot Extreme with Nominal Setpoints

< Still working out kinks in model, Will be added for final submission>

Figure 12 - TECFIRE Prediction for Hot Extreme with Recommended Setpoints 
VII. Conclusions

The flight correlation efforts yielded some updates to the detailed thermal model and justification of the environments used. Overall, many of the adjustments were minor, but the increase cooling demands of TECFIRE as a result of lower environmental flux boundary conditions made them appear much larger at first glance. The empirical model provides confidence in the design based on the flight data obtained to date. Based on correlation to flight data and agreement with the empirical model, the detailed model can now be used to predict thermal performance under worst case hot conditions and performance for key temperature control setpoint changes. These setpoint change recommendations could be implemented to mitigate any potential conditions that could result in loss of detector temperature control and consequently instrument performance.

During the correlation efforts, some lessons were learned that may be applied to other missions with similar designs. While some of the lessons learned were based on experiences with Thermo Electric Coolers, the same philosophy follows for any active cooling device whose power increases with temperature, including TECs and cryocoolers. With the ever decreasing desired detector temperatures and the increased difficulty of achieving this passively, the lessons learned with respect to the effects of environmental loading uncertainty should apply equally, stressing the importance of design margin to account for these uncertainties. These lessons learned are again summarized in the list below:

- Additional test opportunities can be extremely valuable since additional information can be gained from areas using additional test instrumentation that were not known ahead of time to have large uncertainties.

- Model changes made during a correlation effort should never invalidate the correlation to test data from previous testing for regions where the design was not significantly varied.

- Subsystem qualification tests, particularly for thermally sensitive subsystems, can provide a great opportunity for the thermal engineer to gain additional data that may provide confidence in hand or empirical checks at later points in a program.

- For actively cooled systems, the characterization of all heat paths to the hot side, especially environmental heat loads, is of crucial importance to being able to accurately predict a steady condition.

- Once a test correlation is complete, some benefit may be derived by performing a sensitivity study on the tuned parameters. This could identify the range to which these may be further altered for flight correlation without negatively impacting a test correlation.

- When correlating to flight data, care must be taken when selecting the environmental parameters to use. While extreme bounding cases may be the simple choice, they may not be accurate for a specific set of conditions related to flight.

- If a design is very sensitive to environmental parameters and a flight correlation is essential, it may be beneficial to include some means of measuring the environmental fluxes at the radiator in flight.

- Localized optical property effects (holes, cap nuts, etc.) due to mechanical features may artificially influence the sensed temperature values from telemetry. These effects should be considered when temperature data is compared to model predictions.

- Although some surfaces may not be included in a flight model due to a lack of telemetry or insight, their backloading effect on other surfaces should be considered before excluding them from the model.

- Simplistic empirical models based on flight data and energy balances can be a valuable tool to cross check more computationally demanding detailed thermal models.

\section{Acknowledgments}

I would like to thank all the members of the WFC3 team, past and present, who contributed their time and efforts to making WFC3 such a successful instrument. The images and end results of their work is truly breathtaking.

\section{References}

$<$ Will be added for final submission $>$ 